\title{
B amyloid angiogenic activity in vitro and in vivo
}

\author{
ELISA BOSCOLO ${ }^{1}$, MARCELLA FOLIN $^{1}$, BEATRICE NICO $^{2}$, CLAUDIO GRANDI ${ }^{3}$, \\ DOMENICA MANGIERI ${ }^{2}$, VITO LONGO ${ }^{2}$, RENATO SCIENZA ${ }^{4}$, PAOLO ZAMPIERI ${ }^{4}$, \\ MARIA TERESA CONCONI ${ }^{3}$, PIER PAOLO PARNIGOTTO ${ }^{3}$ and DOMENICO RIBATTI ${ }^{2}$ \\ ${ }^{1}$ Department of Biology, University of Padua, Padua; ${ }^{2}$ Department of Human Anatomy and Histology, \\ University of Bari Medical School, Bari; ${ }^{3}$ Department of Pharmaceutical Sciences, \\ University of Padua; ${ }^{4}$ Department of Neurosurgery, Padua Hospital, Padua, Italy
}

Received April 26, 2006; Accepted July 4, 2006

\begin{abstract}
Angiogenesis has been suggested as a direct contributor to Alzheimer's disease (AD) pathology. The major pathological hallmarks of AD are the presence of neurofibrillary tangles and, $B$-amyloid plaques associated with activated microglia, astrocytes, degenerating neurons and vascular toxicity. In this study, $A ß 1-40$ and $A ß 1-42$ peptides, both components of the senile plaques in $\mathrm{AD}$, were used to study their angiogenic activity in vitro, by using normal human cerebral endothelial cells (HCECs), and in vivo, by using the chick embryo chorioallantoic membrane (CAM) assay. Results showed that both peptides stimulate in vitro endothelial cell proliferation, chemotaxis and morphogenesis in Matrigel. Moreover, by using the aorta ring assay, both peptides stimulated the formation of capillary-like structures. An angiogenic response was induced in the CAM assay, similar to that induced by fibroblast growth factor-2 (FGF-2), a well-known angiogenic cytokine. Overall, these data support the hypothesis that $A ß$ peptides may contribute to angiogenesis occurring in $\mathrm{AD}$ and suggest that limiting the pro-angiogenic activity of $\mathrm{A} \beta$ peptides may therefore provide a useful target to control angiogenesis associated to AD and therefore limit the disease progression.
\end{abstract}

\section{Introduction}

Angiogenesis is a complex process, where several cell types and mediators interact to establish a specific microenvironment suitable for the formation of new vessels. Angiogenesis takes place in various physiological and pathological conditions, such as embryonic development, chronic inflammation and tumors (1).

Correspondence to: Dr Domenico Ribatti, Department of Human Anatomy and Histology, University of Bari Medical School, Policlinico, Piazza Giulio Cesare 11, I-70124 Bari, Italy

E-mail: ribatti@anatomia.uniba.it

Key words: Alzheimer's disease, angiogenesis, beta amyloid
Angiogenesis has been suggested as a contributor to Alzheimer's disease (AD) pathology, which is characterized by an increase in microvascular density in the pathological lesions (2). Vascular endothelial growth factor (VEGF), one of the most potent pro-angiogenic factors identified thus far, is implicated in the generation of neo-vessels in almost all types of pathological settings (3), including $\mathrm{AD}$ (4). In fact, increased VEGF expression is seen in reactive astrocytes and perivascular deposits of AD patients (5). Moreover, Tarkowski et al (6) observed significantly increased cerebrospinal fluid levels and intrathecal synthesis of both VEGF and of another angiogenic cytokine, namely transforming growth factor $\beta$ (TFG- $\beta$ ). Pogue and Lukiw (7) showed that VEGF mRNA is upregulated in AD hippocampus 2.7-fold over controls.

Also inflammation may contribute to the angiogenic activity occurring in AD. Elevated neural VEGF levels in humans suffering AD may be secondary to inflammation (6) and, in turn, inflammatory mediators found in the AD brain, such as tumor necrosis factor $\alpha(\mathrm{TNF}-\alpha)$, interleukin-6 (IL-6) and monocyte chemoattractant protein (MCP-1) exert a proangiogenic activity.

Endogenous angiogenesis inhibitors under physiologic conditions may act to limit or prevent angiogenesis. The gene expression of one of these inhibitors, namely thrombospondin, is reduced near focal AD lesions (8) and recent evidence has also been presented indicating that a number of pharmacological agents able to reduce the risk of $\mathrm{AD}$, show anti-angiogenic effects in vitro, ex-vivo, and in vivo in relatively short-term experiments (2).

The major pathological hallmarks of $\mathrm{AD}$ are the presence of neurofibrillary tangles and $\beta$-amyloid plaques associated with activated microglia, astrocytes, degenerating neurons (9) and vascular toxicity. Ultrastructural studies have shown that brain microvessels are closely associated with B-amyloid plaques, and that Alzheimer's disease brain capillaries contain preamyloid deposits (10). The B-amyloid plaque generates reactive oxygen species that damage the brain endothelium (11). Progressive deposition of amyloid precursor protein leads to the accumulation of the $\beta$-amyloid plaque, which generates more reactive oxygen species and induces further endothelial damage.

Amyloid precursor protein (APP) is a ubiquitous transmembrane protein. APP is cleaved by $\alpha$ - or $\beta$-secretases, 
releasing the $100 \mathrm{kDa}$ ectodomain of APP and the 10-12 $\mathrm{kDa}$ membrane-bound C-terminal fragment. An additional cleavage in the transmembrane domain by a $\gamma$-secretase results in secretion of amyloid $B(A \beta)$ peptides (12). $A ß$ peptides are naturally present in low nanomolar quantities as circulating soluble monomers in the blood of healthy individuals (13).

$A ß 1-40$ and $A ß 1-42$ peptides, both components of the senile plaques in $\mathrm{AD}$, were tested to investigate their angiogenic activity in vitro, by using for the first time normal human cerebral endothelial cells (HCECs) and in vivo, in the chick embryo chorioallantoic membrane (CAM) assay, using the fibroblast growth factor-2 (FGF-2), a well-known angiogenic cytokine, as a positive control.

\section{Materials and methods}

Isolation and culture of HCECs. Microvascular HCECs were isolated from fresh biopsies of non-Alzheimer human brain. The samples were obtained from surgical procedures on deep seated lesions. Informed consent of the five patients (aged $30-40$ years) was obtained and the protocol was approved by the local ethics committee. Tissue was sectioned into $3 \mathrm{~mm}^{2}$ fragments, washed with PBS, and incubated in $0.1 \%$ collagenase B/0.1\% dispase (Roche, Basel, Switzerland) at $37^{\circ} \mathrm{C}$ for $25 \mathrm{~min}$. The pre-digested tissue was triturated by a $2 \mathrm{ml}$ pipette for $2 \mathrm{~min}$ and then filtered through a $100 \mu \mathrm{m}$ strainer (BD Biosciences, Franklin Lakes, NJ, USA). Cell suspension was centrifuged and re-suspended in culture medium MV2 supplemented with growth factors and $20 \%$ foetal bovine serum (FBS) (PromoCell, Heidelberg, Germany). Cells were then seeded at a density of $10^{4}$ cells $/ \mathrm{cm}^{2}$ on Petri dishes coated with fibronectin $\left(1 \mu \mathrm{g} / \mathrm{cm}^{2}\right)$ (Sigma Aldrich Inc., St. Louis, $\mathrm{MO}, \mathrm{USA}$ ) and grown for $24 \mathrm{~h}$ at $37^{\circ} \mathrm{C}$ with $5 \% \mathrm{CO}_{2}$, washed vigorously with $\mathrm{PBS}$ to remove unattached cells, and fed with fresh MV2/20\% FBS. HCECs were purified from $80-100 \%$ confluence primary culture using Ulex europaeus agglutinin I (UEA)-coated (Vector Laboratories, Ltd., Peterborough, UK) Dynabeads M-450 Tosylactivated (Oxoid, Hampshire, UK), as described by Jackson et al (14). HCECs bound to the lectin-coated beads were collected with a magnetic particle concentrator, and any unbound cells were removed with two washes with basal medium and re-suspended in culture medium. Cells were seeded on fibronectin-coated Petri dishes to improve their adhesion and growth. Cultures became confluent within 5-8 days.

Characterization of HCECs. Immunocytochemistry was performed to characterize the isolated cells with rabbit polyclonal anti-human von Willebrand factor (vWF) (diluted 1:300; Dako Corp., Carpinteria, CA, USA) For immunostaining, cells were grown in MV2/20\% FBS on fibronectincoated chamber slides, washed in PBS, and fixed in ice-cold aceton for $10 \mathrm{~min}$ at $4^{\circ} \mathrm{C}$. Immunostaining was performed at room temperature, and cells were washed three times in PBS after each step. PBS with $1 \%$ bovine serum albumin (BSA) was used for antibody dilution. Cells were treated with $1 \%$ Triton in PBS for $5 \mathrm{~min}$ at room temperature and then incubated at room temperature with $1 \%$ BSA in PBS for 15$20 \mathrm{~min}$ to block non-specific binding sites. The primary antibody rabbit anti-human vWF (1:300) was added, for $1 \mathrm{~h}$, and then the secondary peroxidase-conjugated anti-rabbit IgG (1:150) for $30 \mathrm{~min}$. The reaction was developed with avidin-biotin amplified immunoperoxidase method, using the Large Volume Dako LSAB Peroxidase kit (Dako, Glostrup, Denmark). Finally, samples were counterstained with hematoxylin. Negative controls were performed by omitting the primary antibody. Slides were mounted with Eukitt (Fluka, Buchs, Switzerland).

Cell proliferation assay. HCECs $\left(3 \times 10^{3}\right)$ re-suspended in MV2/20\% FBS were seeded in each well of 96-multiwell plates. After adherence (5-6 h), the supernatant was replaced with medium without growth factors containing $0.1 \% \mathrm{BSA}$, and cells were incubated with either rat Aß1-40, Aß1-42 or reverse sequence peptides $\mathrm{A} \beta 40-1$ and $\mathrm{A} \beta 42-1$ (1 and $100 \mathrm{nM})$ (Calbiochem, Darmstatdt, Germany). The two concentrations were chosen because previously it was demonstrated that Aß1-40 peptide at nanomolar concentrations stimulated endothelial cell growth, while when it was tested at micromolar concentrations, inhibited endothelial cell growth and caused cell death (15). Peptides were solubilized accordingly to the manifacturer's instructions.

After 24-48 h, proliferation was measured using BrdU incorporation (Cell Proliferation ELISA, BrdU, Roche Applied Science, Monza, Italy). Briefly, BrdU labeling solution was added to the medium and incubated in a $5 \% \mathrm{CO}_{2}$ incubator at $37^{\circ} \mathrm{C}$ for $18 \mathrm{~h}$. The culture medium was removed after the labeling step and the FixDenat (cell fixation and DNA denaturation) reagent was added. After $30 \mathrm{~min}$ of incubation at room temperature, the FixDenat was removed. Subsequently, cells were incubated with anti-BrdU-POD antibody conjugate for $1 \mathrm{~h}$. The plates were then washed before the substrate was added. The absorbance was determined at $405 \mathrm{~nm}$ wavelength, with a reference wavelength of $490 \mathrm{~nm}$, with a microplate autoreader EL13. The means of four experiments were expressed as percent change from cultures seeded without peptides.

Cell migration. Chemotaxis experiments were performed with the Boyden chamber technique (24-well chemotaxis chamber) using $5 \mu \mathrm{m}$ pore size polycarbonate membranes (Costar, Acton, MA, USA). HCECs $\left(4 \times 10^{4}\right)$ were seeded in $100 \mu 1$ medium into the upper surface of the membrane insert. To assess the pro-migratory effect either rat AB1-40, Aß1-42 or reverse sequence peptides Aß40-1 and Aß42-1 (1 and $100 \mathrm{nM}$ ) were placed in the lower compartment of the chamber. After incubation at $37^{\circ} \mathrm{C}$ for $4 \mathrm{~h}$, the upper surface of the filter was gently scraped to remove non-migrated cells. Filters were fixed with $4 \%$ formaldehyde and stained with 4',6-Diamidino-2-phenylindole (DAPI) (Vector Laboratories, Ltd.). Experiments were performed in duplicate and the number of cells present in 5 fields per well was counted at $\times 10$ magnification in a blinded manner using a Laborlux-S microscope (Leitz, Wetzlar, Germany).

Morphogenesis in Matrigel. We also checked purified HCECs for their ability to form capillary-like structures. Briefly, growth factor-reduced Matrigel (BD Biosciences) was thawed on ice overnight and spread evenly over each well $(50 \mu 1)$ of a 24 -well plate. The plates were incubated for 


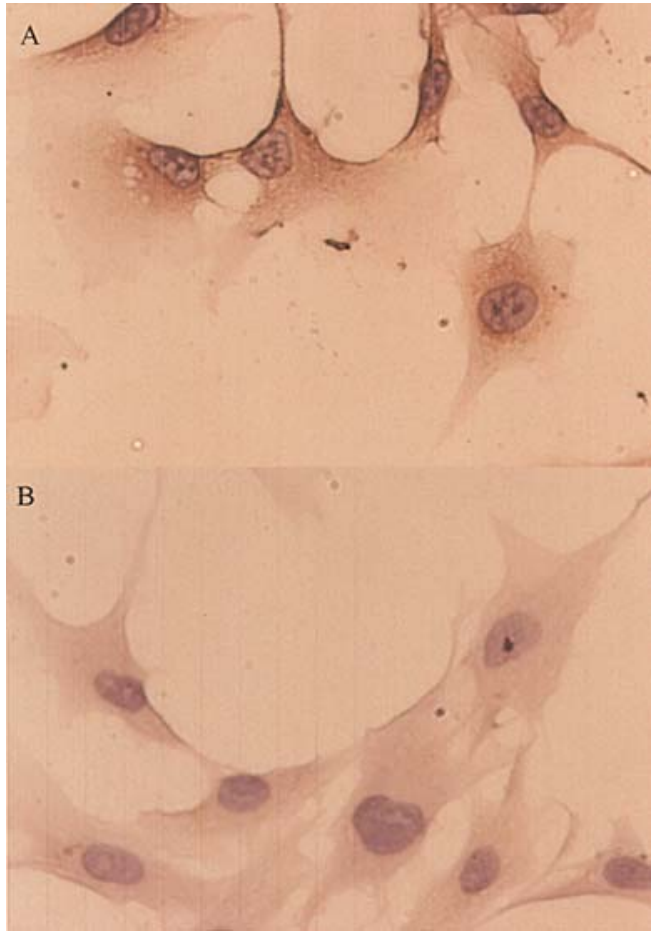

Figure 1. Immunocytochemical staining of human cerebral endothelial cells. Purified HCECs stained positively for von Willebrand Factor (vWF) antibody showing a typical punctate brown immunoreaction (A), not detectable when the primary antibody is omitted (B). Nuclei are counterstained with hematoxylin. Original magnifications: A and B, x50.

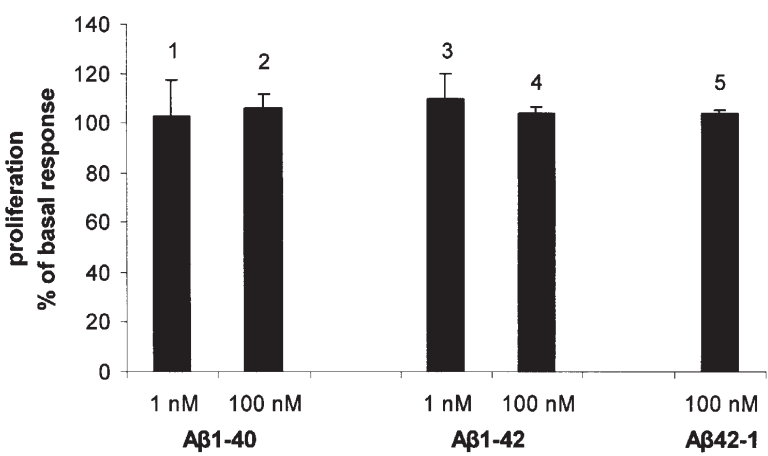

A

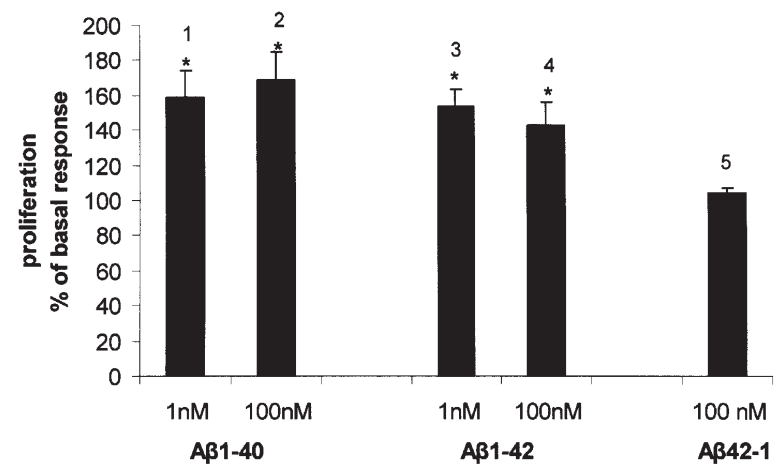

B

Figure 2. Aß peptides promote endothelial cell proliferation at $48 \mathrm{~h}$. Growth of HCECs exposed to 1 and $100 \mathrm{nM}$ concentrations of Aß1-40 or Aß1-42 peptides and to reverse sequence peptide Aß42-1 $100 \mathrm{nM}$ after $24 \mathrm{~h}$ (A) and $48 \mathrm{~h}(B)$ of incubation. Data are percentage of basal response $\pm \mathrm{SD}(\mathrm{n}=4$ experiments). ${ }^{*} \mathrm{p}<0.05$ vs control. Statistical analysis was performed by ANOVA.

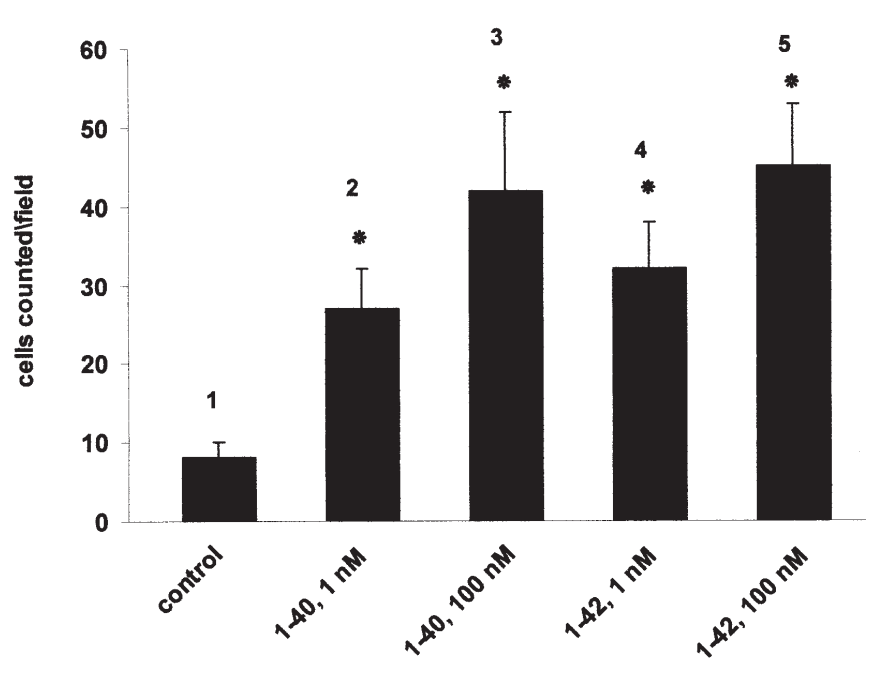

Figure 3. Aß peptides promote endothelial cell migration. HCECs are induced to migrate toward $A ß 1-40$ and $A ß 1-42$ peptides tested at 1 and $100 \mathrm{nM}$. Migrated cells counted for each field are higher than cells counted in the control (Aß42-1 $100 \mathrm{nM})$. Error bars represent SD $(\mathrm{n}=5$ field for two experiments). ${ }^{*} \mathrm{p}<0.05$ vs control. Statistical analysis was performed by ANOVA.

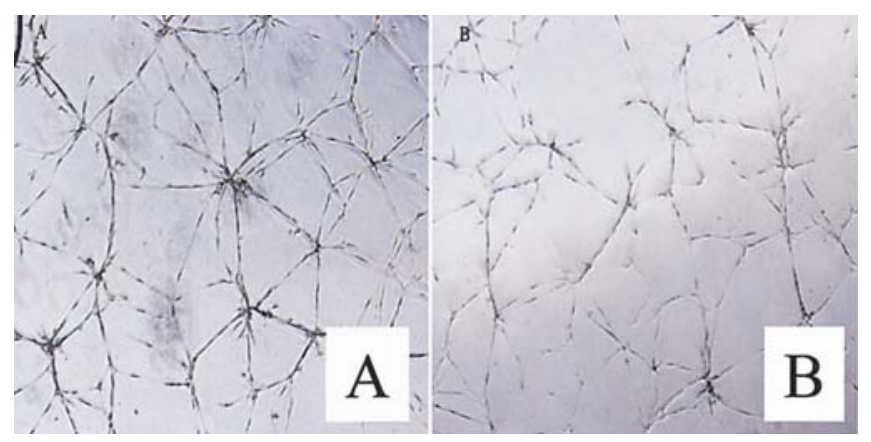

Figure 4. Aß peptide-induced tube formation by HCECs seeded on Matrigel. Representative photographs of HCEC cultures on Matrigel after incubation for $48 \mathrm{~h}$ with $100 \mathrm{nM} \mathrm{A} 31-42$ peptide (A) and reverse sequence peptide Aß42-1 (B). Original magnifications: A and B, x5.

$20 \mathrm{~min}$ at $37^{\circ} \mathrm{C}$ to allow the Matrigel to gel. HCECs $\left(2.5 \times 10^{4}\right.$ cells $/ \mathrm{cm}^{2}$ ) were seeded into each well in $500 \mu 1$ of MV2/0.1\% $\mathrm{BSA}$ medium and incubated for $24-48 \mathrm{~h}$ at $37^{\circ} \mathrm{C}$. Either $\mathrm{A} ß 1-$ $40, \mathrm{~A} ß 1-42$ or reverse sequence peptides $\mathrm{A} ß 40-1$ and $\mathrm{A} ß 42-1$ ( 1 and $100 \mathrm{nM}$ ) were added to check their ability to promote tubular structure formation. Experiments were performed in duplicate. Cultures were fixed with $10 \%$ paraformaldehyde for $10 \mathrm{~min}$. After rinsing with PBS, cultures were observed by phase contrast microscopy (Leitz Laborlux S microscope). Cultures were photographed (five random fields chosen in a blinded manner for each well) at x 5 magnification. Phase contrast images were recorded using a digital camera (DG 200; Leica Imaging System, Cambridge, UK) connected to a Laborlux-S microscope. Image analysis was carried out using the Qwin image analysis software (Leica Imaging System), and the dimensional (percent area covered by cells and the total length of cell network per field), and topological 
A

24h: dimensional parameters

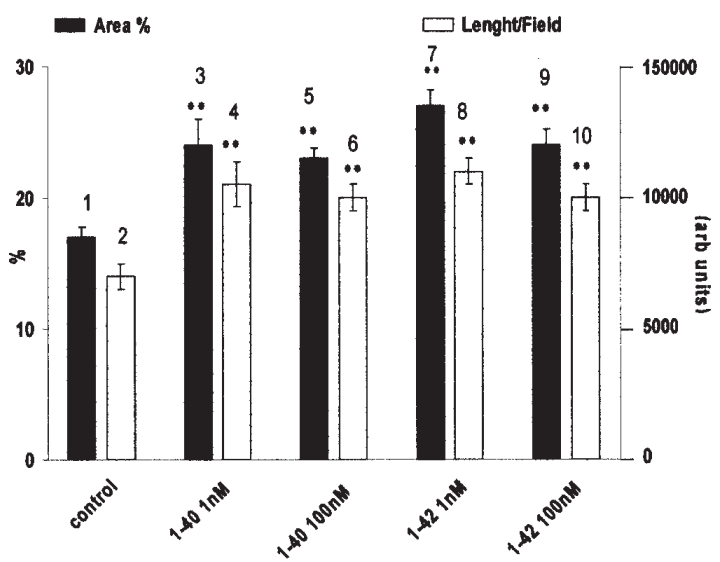

C

48h: dimensional parameters

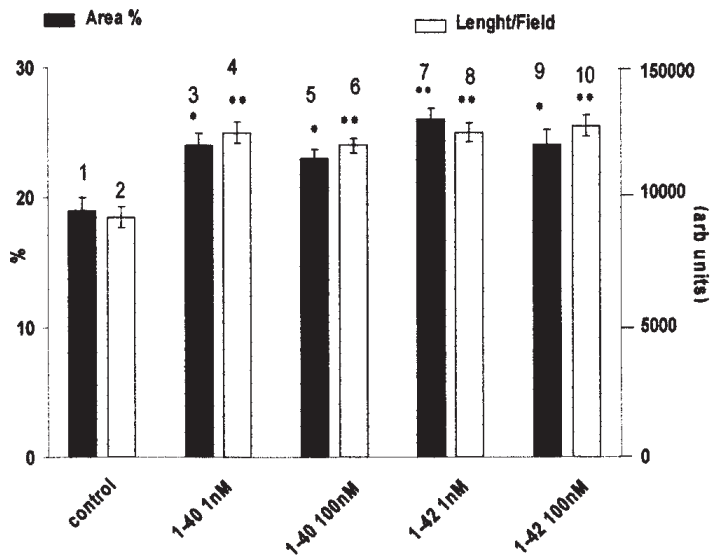

B 24h: topological parameters

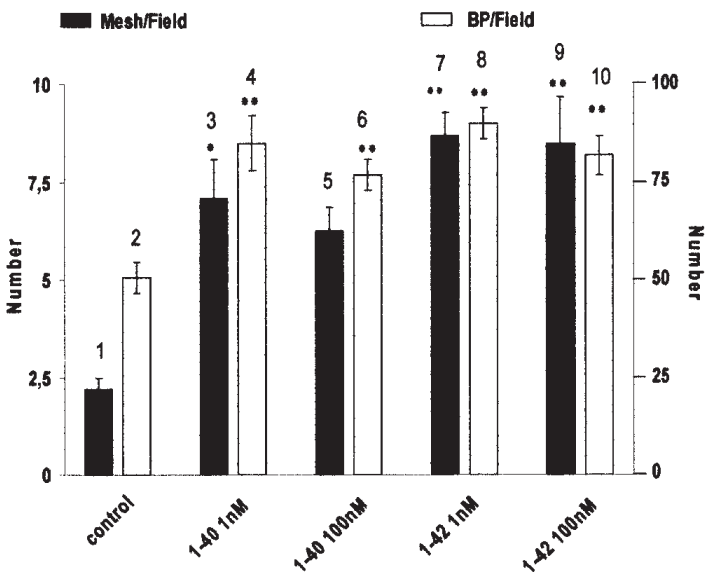

D

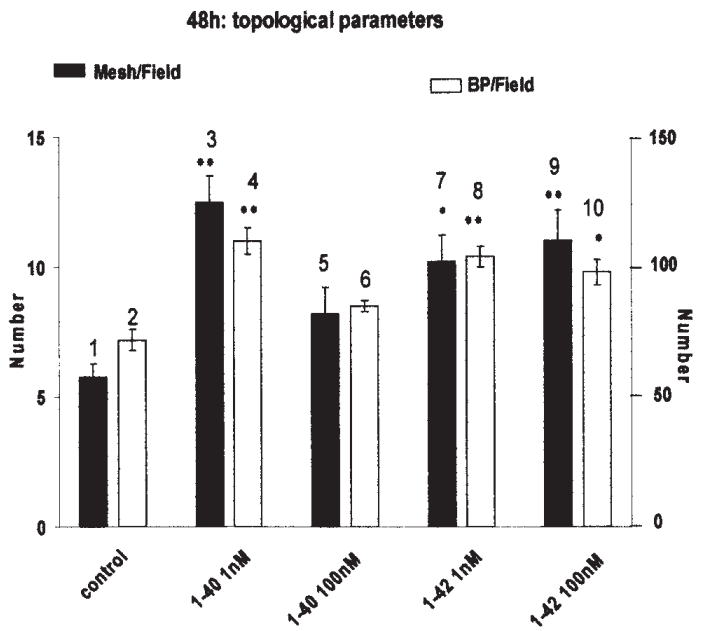

Figure 5. Quantitative analysis of tube formation. Quantitative evaluation, estimated as dimensional (A and C, percent area covered by cells and total length of cell network per field) and topological (B and D, mesh number and branching points per field) parameters indicate that both peptides after $24-48 \mathrm{~h}$ of incubation produce an increase in tube formation at both 1 and $100 \mathrm{nM}$ concentration compared with the reverse sequence peptide (control). Each column represents mean $\pm \mathrm{SD}$ of 5 different fields for 2 independent experiments compared with control; * $\mathrm{p}<0.05$, ** p<0.01 vs control. Statistical analysis was performed by ANOVA and Dunnet's test.

parameters (mesh number and branching points per field) were estimated and used to characterize the topological structure of endothelial cells accordingly to Guidolin et al (16).

Endothelial tube formation from aortic rings. Briefly, 1-2 mm vessel rings obtained from rat aorta (1-12 months old) were positioned in 24-multiwell plates with the lumen oriented horizontally. $30 \mu 1$ of Matrigel onto each aorta section were spread and the organ culture was kept 20 min at $37^{\circ} \mathrm{C}$ in $5 \%$ $\mathrm{CO}_{2}$ to allow the Matrigel to solidify. Stimuli were tested adding to the medium supplemented with $1 \%$ BSA either $\mathrm{A} \beta 1-40, \mathrm{~A} \beta 1-42$ or reverse sequence peptides $\mathrm{A} \beta 40-1$ and Aß42-1 (1 and $100 \mathrm{nM}$ ). The medium with test substances was replaced every 3 days. Response to $\mathrm{MV} / 1 \% \mathrm{BSA}$ alone was also evaluated. After 12 days, pictures were taken with a Labolux-S microscope.

Statistical analysis. Values are presented as mean \pm standard deviation (SD). Data within each group were compared by ANOVA for repeated measurements, and if significant, a post hoc comparison by paired t-test was performed. Significance was defined as $\mathrm{p}<0.05$. Statistical analysis was performed using Primer software.

CAM assay and measurement of angiogenesis in vivo. Fertilized White Leghorn chicken eggs (30 for each series) were incubated under conditions of constant humidity at $37^{\circ} \mathrm{C}$. On day 3 of incubation, a square window was opened in the shell after removal of 2-3 ml of albumen to allow detachment of the developing CAM. The window was covered with a transparent glass of the same dimension, sealed with paraffin, and then the eggs were returned to the incubator.

In a first series of experiments, on incubation day 8 , under sterile conditions within a laminar flow hood, CAMs were implanted with $1-\mathrm{mm}^{3}$ sterilized gelatine sponges (Gelfoam; Upjohn Co, Kalamazoo, MI, USA) (17) loaded with $50 \mathrm{ng}$ /embryo of $\mathrm{A} \beta 1-40$ or $\mathrm{A} \beta 1-42$ dissolved in $1 \mu 1$ PBS. CAMs implanted with sponges loaded with PBS alone or with $500 \mathrm{ng} / \mathrm{embryo}$ of human recombinant FGF-2 (R \& D Systems, Abindgton, UK) were used as negative and positive 

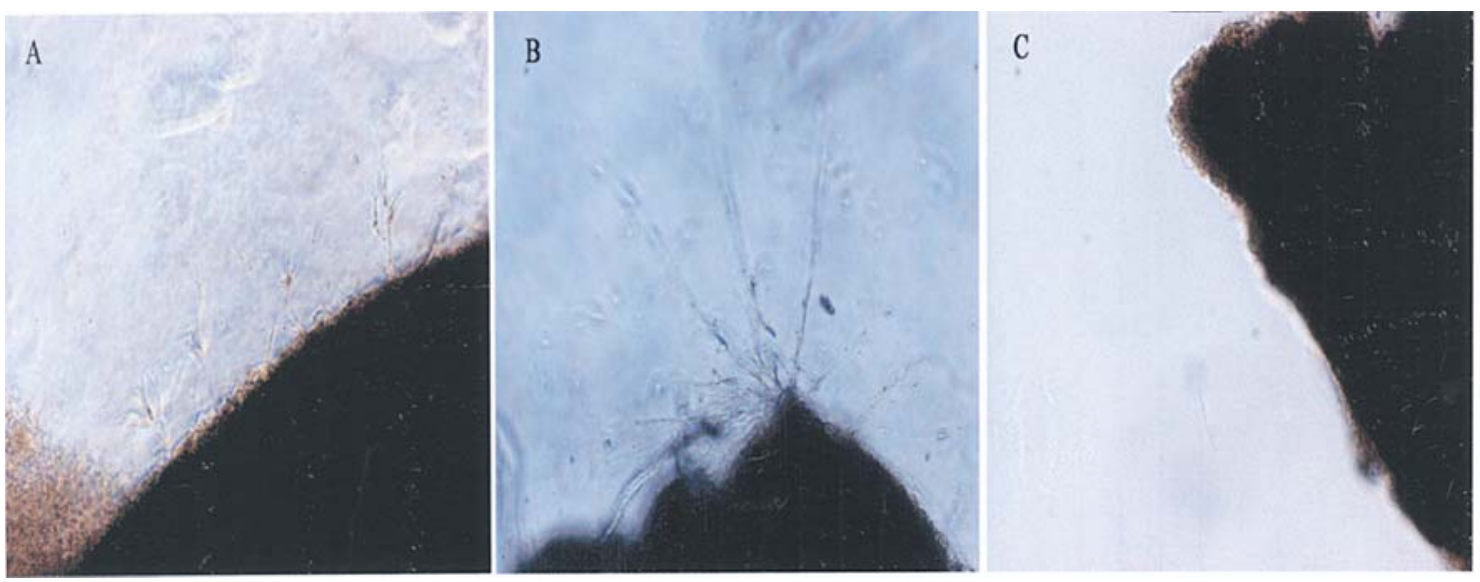

Figure 6. Effect of $A ß$ peptides on new vessel formation in a rat aorta explant model. Capillary sprouting from rat aorta rings at day 12 after administration of $100 \mathrm{nM} \mathrm{Aß1-40} \mathrm{(A)} 100 \mathrm{nM}$ Aß1-42 (B) and after administration of reverse sequence peptide Aß42-1 (C). Original magnifications: A-C, x10.
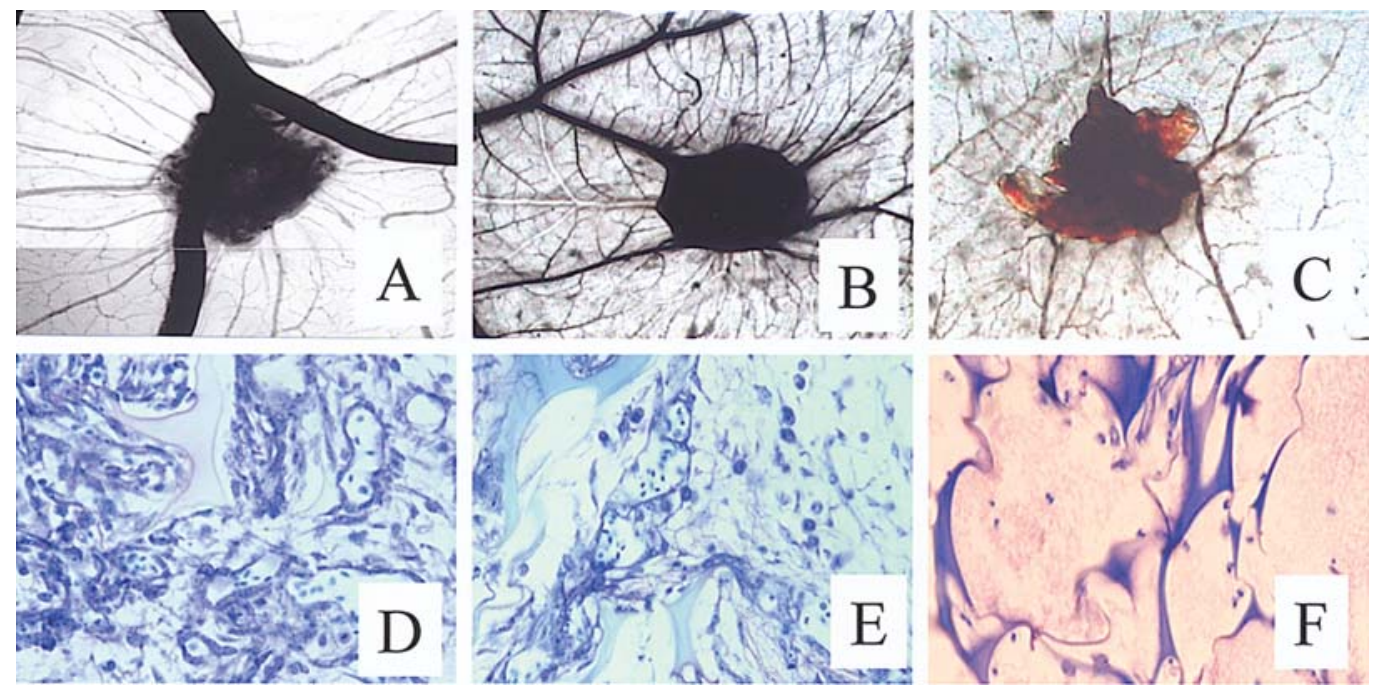

Figure 7. Angiogenic activity of $A B$ peptides in vivo in the CAM assay. (A and B) CAMs at day 12 of incubation, $96 \mathrm{~h}$ after the $A B 1-40$ (A) or $A B 1-42$ (B) peptide-treated sponges were implanted. Note the presence of numerous blood vessels converging toward the implants. (C) In contrast, no vascular response is detectable around the control implant. (D, E and F) Histological pictures of CAMs treated with Aß1-40 (D), Aß1-42 (E) peptides and with PBS (F). Note in $\mathrm{D}$ and $\mathrm{E}$, numerous microvessels inside the sponge, while in the control specimen (F) no blood vessels are recognizable. Original magnifications: A-C, $\mathrm{x} 50$; D-F, $x 250$.

controls, respectively. CAMs were examined daily until day 12. Then the CAMs were processed for light microscopy: after the embryos and their membranes were fixed in ovo in Bouin's fluid, the sponges and the underlying and immediately adjacent CAM portions were removed, processed for embedding in paraffin. $8 \mu \mathrm{m}$ serially-sections cut along a plane parallel to the CAM surface were stained with $0.5 \%$ toluidine blue and observed under a Zeiss Axiophot-2 light microscope.

Angiogenesis was measured in the sponges by a planimetric 'point counting' method (18) for the computerized image analysis (KS 300 software, Zeiss). Four to six x250 fields covering almost the entire field of every third section within 30 serial sections of at least two sponges per sample were analysed with a superimposed 144-point square reticulum $\left(0.0186 \mathrm{~mm}^{2} /\right.$ field and $129.13 \mu \mathrm{m}^{2} /$ point $)$ to determine the number of microvessels (capillaries and post-capillary venules) both inside the sponge and at the boundary between the sponge and the surrounding CAM mesenchyme. The microvessel area was then measured within the reticulum area as the sum of points that hit microvessels, and recorded as the mean percentage \pm SD per sponge and sample. The statistical significance of the differences between mean values was determined by the Students t test for unpaired data.

\section{Results}

Characterization of isolated HCECs. To verify that the isolated Ulex europaeus I bead-bound cells were indeed endothelial cells and free from contaminating non endothelial cells, expression of endothelial-specific marker vWF was investigated by immunocytochemistry. HCECs exhibited punctate cytoplasmic staining with anti-vWF (Fig. 1A), 
Table I. Quantification of angiogenic response in the CAM assay at microscopic level.

\begin{tabular}{lcc}
\hline Specimens & Microvessel density $\%^{\mathrm{a}}$ & Intersection points $^{\mathrm{b}}$ \\
\hline Aß1-40 & 19.4 & $28 \pm 4^{\mathrm{c}}$ \\
Aß1-42 & 18.0 & $26 \pm 3^{\mathrm{c}}$ \\
PBS & 4.2 & $6 \pm 2$ \\
FGF-2 & 20.8 & $30 \pm 5^{\mathrm{c}}$ \\
\hline
\end{tabular}

aSee Materials and Methods for further details. ${ }^{b}$ Mean \pm SD. ${ }^{c} p<0.001$ vs PBS implants. The statistical significance of the differences between mean values was determined by the Student's t test for unpaired data.

consistent with localization of vWF in Weibel- Palade bodies, a typical feature of endothelial cells. No background staining was observed omitting the primary antibody (Fig. 1B). Cells at passage 3-5 that stained $95 \%$ positively for vWF were used.

Aß peptides promote endothelial cell growth, migration, and tube formation in vitro. First, the effect of $\mathrm{A} B$ peptides on endothelial cell proliferation was assessed. Exposure of quiescent HCECs to both $\mathrm{A} \beta$ peptides, tested at nanomolar concentrations ( 1 and $100 \mathrm{nM}$ ), induced them to proliferate following 48-h exposure (Fig. 2B, lines 1-4) but any proliferative response was seen at $24 \mathrm{~h}$ (Fig. 2A, lines 1-4). At the concentration tested no toxic effects were detectable, accordingly to Cantara et al (15). The reverse sequences Aß40-1 and Aß42-1 did not affect endothelial cell growth neither at 24 nor at $48 \mathrm{~h}$ (Fig. 2A and B, lines 5). In all the following experiments no significative difference was detected comparing response to reverse peptides (at both 1 and $100 \mathrm{nM}$ ) and response to $\mathrm{MV} / 0.1 \% \mathrm{BSA}$ medium alone. For this reason results were simplified using Aß42-1 $100 \mathrm{nM}$ as control.

Migration is another parameter of pro-angiogenic activity. In all the induction experiments, when cells were exposed to $\mathrm{A} ß 1-40$ and $\mathrm{A} ß 1-42$ peptides tested at 1 and $100 \mathrm{nM}$ concentration, a 3 to 6 -fold increase in the number of migrating cells was detectable (Fig. 3, lines 2-5), as compared with the reverse sequence peptide Aß42-1 $100 \mathrm{nM}$ (Fig. 3, line 1).

To determine whether $A B$ peptides have angiogenic potential in vitro, capillary-like tube formation was assessed by seeding HCECs on growth factor-reduced Matrigel. As shown in Fig. 4A, the $48 \mathrm{~h}$ treatment of HCECs with $100 \mathrm{nM}$ Aß1-42 peptide led to a strong induction of tube formation, as compared to cells cultured in the presence of the reverse sequence peptide (Fig. 4B).

Quantitative analysis indicates that both peptides after 24$48 \mathrm{~h}$ of incubation, tested at 1 and $100 \mathrm{nM}$ induced an increase in dimensional parameters, such as percent area covered by cells (Fig. 5A and C, lines 3, 5, 7, 9) and the total length of cell network per field (Fig. 5A and C, lines 4, 6, 8, 10) and topological parameters, such as mesh number (Fig. 5B and D, lines $3,5,7,9$ ) and branching points per field (Fig. 5B and D, lines $4,6,8,10)$ as compared with the reverse sequence peptide (control) (Fig. 5A-D, lines 1 and 2).

Afterwards, $A ß$ peptides were tested in the ex vivo formation of microvessel network from rat aorta explants.
When tested at a $100 \mathrm{nM}$ concentration both peptides allowed endothelial tube sprouting from aorta rings (Fig. 6A and $\mathrm{B}$ ), while, no tubes formation was recognizable omitting peptide treatment and in the control specimens (Fig. 6C).

$A \beta$ peptide angiogenic activity in vivo in the CAM assay. By using the CAM assay, on day 12 of incubation, macroscopic examination showed that sponges treated with both AB1-40 and Aß1-42 peptides were surrounded by numerous allantoic vessels, which developed radially towards the implant in a spoke wheel pattern (Fig. 7A and B). A similar picture was produced by sponges loaded with FGF-2 (not shown). No vascular response was detected around the sponges treated with PBS alone (Fig. 7C).

Histological examination of the sponges treated with both $\mathrm{A} ß 1-40$ and $\mathrm{A} \beta 1-42$ peptides showed numerous small blood vessels inside the sponge trabeculae (Fig. 7D and E). Moreover, at the boundary between the sponge and the CAM mesenchyme, numerous host capillaries were recognizable, piercing the sponge at certain points. In contrast, no blood vessels were recognizable among the sponge trabeculae in the sponges treated with PBS (Fig. 7F). Table I shows microvessel counts $96 \mathrm{~h}$ after sponge implants, which confirm the macroscopic observations. There was a statistically significant difference between Aß1-40/Aß1-42 peptides- and PBS-loaded specimens in microvessel counts in the CAM area under the implant. The angiogenic response induced by both $A ß 1-40$ and $A ß 1-42$ peptide implants was comparable to that obtained with 500 ng FGF-2.

\section{Discussion}

Angiogenesis is a complex process characterized by a cascade of events including activation and migration of endothelial cells, the degradation and the remodeling of basement membrane and surrounding extracellular matrix, endothelial cell proliferation, and neovessel formation (1). The process is activated by the synthesis and release of angiogenic factors and/or switching off of anti-angiogenic factors by the cells (1).

Two major possibilities have been advanced to explain the source of $\beta$ amyloid in the amyloid plaque: the neuronal and vascular explanation. The vascular hypothesis rests mainly on the deposition of amyloid in the walls of blood vessels in $\mathrm{AD}$ and on the intimate association of capillaries with amyloid plaques. This hypothesis is supported by the evidence that angiogenesis occurs in AD brain (19) and that endothelial cell proliferation and migration are likely to be important factors in the progression of amyloid angiopathy in $\mathrm{AD}$ brain.

Herein, it has been demonstrated, using for the first time normal human cerebral endothelial cells, that Aß1-40 and Aß1-42 peptides, both components of senile plaques in $\mathrm{AD}$, stimulate in vitro the angiogenic phenotype, which is characterized by certain cell functions, such as proliferation, chemotaxis and morphogenesis into vascular tubes. These functions are essential for angiogenesis, since proliferation and chemotaxis determine sprouting of the microvessel, whereas morphogenesis leads to its stabilization and functional maturity.

These theories have been confirmed by using the aorta ring assay, an ex-vivo model of angiogenesis, in which endo- 
thelial cells and periendothelial cells, such as vascular smooth muscle cells and pericytes, and the extracellular matrix components are involved (20). Here, by using this assay, it was shown that both $A ß 1-40$ and $A ß 1-42$ peptides stimulated human cerebral endothelial cells to organize in capillary-like structures. Additionally, capillary structures induced by $A ß 1-42$ are more elongated than structures seen with $A ß 1-40$ treatment.

To confirm the in vivo relevance of the pro-angiogenic activity of $A ß 1-40$ and $A ß 1-42$ peptides, the chick embryo $\mathrm{CAM}$, an extraembryonic membrane which serves as a gas exchange surface and is supported by an extensive capillary network, and is widely utilized as a target for angiogenic and anti-angiogenic compounds (21), was utilized. Also when tested in this system, Aß1-40 and Aß1-42 peptides significantly increased angiogenesis, similarly to FGF-2, a well-known angiogenic cytokine.

Overall, these data are in accord with those previously published by Cantara et al $(15,22)$, showing that Aß1-40 peptide stimulates endothelial cells to acquire an angiogenic phenotype through interaction with endogenous FGF-2 and induction of its synthesis. Another confirmation to the angiogenic activity of $\mathrm{A} B$ peptides comes from the study of Zand et al (23), which examined neovascularization following injection of AB1-42 peptide into the rat hippocampus. They showed that 7 days following peptide injection neovascularization was markedly up-regulated compared to the control. Moreover, immunoreactivity for the angiogenic cytokine VEGF was also significantly increased and double immunofluorescence staining demonstrated that the increased level of VEGF immunoreactivity was localized to both astrocytes and microglia, suggesting that inflammatory responses contributed to angiogenesis. Finally, A 3 depositis have been found in age-related macular degeneration, where blindness is due to choroidal neovascularization and increased VEGF expression (24).

In summary, the data presented here showed that both $\mathrm{A} ß 1-40$ and $\mathrm{A} ß 1-42$ peptides act directly on endothelial cells, promoting their proliferation, migration, morphogenesis on Matrigel and induction of new vessel formation in the aorta rings and in the CAM assay. The data also support the hypothesis that $A B$ peptides may contribute to angiogenesis occurring in $\mathrm{AD}$ and validate the hypothesis that vascular damage may accelerate the development of AD. Moreover, these results suggest that limiting the pro-angiogenic activity of $A B$ peptides may therefore provide a useful target to control angiogenesis associated to AD and therefore control the disease progression. Nevertheless, further studies are needed to verify if cerebral endothelial cells isolated from AD patients exhibit different phenotypic behaviour.

\section{Acknowledgements}

This work was supported by Associazione Italiana per la Ricerca sul Cancro (AIRC, National and Regional Funds), Milan, Ministry for Education, the Universities and Research (FIRB2001 and PRIN2005) Rome, and Fondazione Italiana per la Lotta al Neuroblastoma, Genoa, Italy. The work has been done on behalf of Veneto Region (Giunta RegionaleRicerca Sanitaria Finalizzata-Venezia, Italy) in collaboration with U.L.S.S. no. 13 of Dolo-Mirano.

\section{References}

1. Folkman J: Angiogenesis in cancer, vascular, rheumatoid arthritis and other diseases. Nat Med 1: 27-31, 1995.

2. Vagnucci AH Jr and Li WW: Alzheimer's disease and angiogenesis. Lancet 361: 605-608, 2003.

3. Ribatti D: The crucial role of vascular permeability factor/vascular endothelial growth factor in angiogenesis: a historical review. Br J Haematol 128: 303-309, 2005.

4. Greenberg DA and Jin K: From angiogenesis to neuropathology. Nature 438: 954-959, 2005.

5. Kalaria RN, Cohen DL, Premkumar DR, Nag S, La Manna JC and Lust WD: Vascular endothelial growth factor in Alzheimer's disease and experimental cerebral ischemia. Brain Res Mol Brain Res 62: 101-105, 1998

6. Tarkowski E, Issa R, Sjogren M, Wallin A, Blennow K, Tarkowski A and Kumar P: Increased intratechal levels of the angiogenic factor VEGF and TGF-beta in Alzheimer's disease and vascular dementia. Neurobiol Aging 23: 237-243, 2002.

7. Pogue AI and Lukiw WJ: Angiogenic signaling in Alzheimer's disease. Neuroreport 15: 1507-1510, 2004.

8. Buee L, Hof PR, Roberts DD, Delacourte A, Morrison JH and Fillit HM: Immunohistochemical identification of thrombospondin in normal human brain and Alzheimer's disease. Am J Pathol 141: 783-788, 1992.

9. Selkoe DJ: Alzheimer's disease: genes, proteins, and therapy. Physiol Rev 81: 741-766, 2001.

10. Miyakawa T: Electron microscopy of amyloid fibrils and microvessels. Ann NY Acad Sci 826: 25-34, 1997.

11. Liu F, Lau BH, Peng Q and Shah V: Pycnogenol protects vascular endothelial cells from beta-amyloid-induced injury. Biol Pharm Bull 23: 735-737, 2000.

12. Haass C, Schlossmacher MG, Hung AY, Vigo-Pelfrey C, Mellon A, Osteszewski BL, Lieberburg I, Koo EH and Schenk D: Amyloid beta-peptide is produced by cultured cells during normal metabolism. Nature 359: 322-325, 1992.

13. Price JM, Chi X, Hellermann G and Sutton ET: Physiological levels of beta amyloid induce cerebral vessels dysfunction and reduce endothelial nitric oxide production. Neurol Res 23: 506-512, 2001.

14. Jackson CJ, Garbett PK, Nissen B and Schrieber L: Binding of human endothelium to Ulex europaeus I-coated Dynabeads: application to the isolation of microvascular endothelium. J Cell Sci 96: 257-262, 1990.

15. Cantara S, Ziche M and Donnini S: Opposite effects of beta amyloid on endothelial cell survival: role of fibroblast growth factor-2 (FGF-2). Pharmacol Rep 57: S138-S143, 2005.

16. Guidolin D, Vacca A, Nussdorfer GG and Ribatti D: A new image analysis method based on topological and fractal parameters to evaluate the angiostatic activity of docetaxel by using Matrigel assay in vitro. Microvasc Res 67: 117-124, 2004.

17. Ribatti D, Gualandris A, Bastaki M, Vacca A, Iurlaro M, Roncali L and Presta M: New model for the study of angiogenesis and antiangiogenesis in the chick embryo chorioallantoic membrane: the gelatin sponge/chorioallantoic membrane assay. J Vasc Res 34: 455-463, 1997.

18. Ribatti D, Urbinati C, Nico B, Rusnati M, Roncali L and Presta M: Endogenous basic fibroblast growth factor is implicated in the vascularization of the chick embryo chorioallantoic membrane. Dev Biol 170: 39-49, 1995.

19. Perlmutter LS, Barron E, Chui HC: Morphologic association between microglia and senile plaque amyloid in Alzheimer's disease. Neurosci Lett 119: 32-26, 1990.

20. Nicosia RF and Ottinetti A: Growth of microvessels in serumfree matrix culture of rat aorta. A quantitative assay of angiogenesis in vitro. Lab Invest 63: 115-122, 1990.

21. Ribatti D, Nico B, Vacca A, Roncali L, Burri PH and Djonov V: Chorioallantoic membrane capillary bed: a useful target for studying angiogenesis and anti-angiogenesis in vivo. Anat Rec 264: 317-324, 2001 .

22. Cantara S, Donnini S, Morbidelli L, Giacchetti A, Schulz R, Memo M and Ziche M: Physiological levels of amyloid peptides stimulate the angiogenic response through FGF-2. FASEB J 18: 1943-1945, 2004.

23. Zand L, Ryu JK and McLarnon J: Induction of angiogenesis in the $B$-amyloid peptide-injected rat hippocampus. Neuroreport 16: $129-132,2005$.

24. Yoshida T, Ohno-Matsui K, Ichinose S, Sato T, Iwata N, Saido TC, Hisatomi T, Mochizuki M and Morita I: The potential role of amyloid beta in the pathogenesis of age-related macular degeneration. J Clin Invest 115: 2793-2800, 2005. 